\title{
Nitrate and nitrite in biology, nutrition and therapeutics
}

Jon O Lundberg, Mark T Gladwin, Amrita Ahluwalia, Nigel Benjamin, Nathan S Bryan, Anthony Butler, Pedro Cabrales, Angela Fago, Martin Feelisch, Peter C Ford, Bruce A Freeman, Michael Frenneaux, Joel Friedman, Malte Kelm, Christopher G Kevil, Daniel B Kim-Shapiro, Andrey V Kozlov, Jack R Lancaster Jr, David J Lefer, Kenneth McColl, Kenneth McCurry, Rakesh P Patel, Joel Petersson, Tienush Rassaf, Valentin P Reutov, George B Richter-Addo, Alan Schechter, Sruti Shiva, Koichiro Tsuchiya, Ernst E van Faassen, Andrew J Webb, Brian S Zuckerbraun, Jay L Zweier \& Eddie Weitzberg

Inorganic nitrate and nitrite from endogenous or dietary sources are metabolized in vivo to nitric oxide (NO) and other bioactive nitrogen oxides. The nitrate-nitrite-NO pathway is emerging as an important mediator of blood flow regulation, cell signaling, energetics and tissue responses to hypoxia. The latest advances in our understanding of the biochemistry, physiology and therapeutics of nitrate, nitrite and NO were discussed during a recent 2-day meeting at the Nobel Forum, Karolinska Institutet in Stockholm.

Until recently the inorganic anions nitrate $\left(\mathrm{NO}_{3}^{-}\right)$and nitrite $\left(\mathrm{NO}_{2}^{-}\right)$were considered inert end products of nitric oxide (NO) metabolism and unfavorable dietary constituents. However, a new view is evolving with the accumulating evidence that nitrate and nitrite metabolism occurs in blood and tissues to form $\mathrm{NO}$ and other bioactive nitrogen oxides, representing an alternative to 'classical' L-arginineNO synthase-NO signaling ${ }^{1-5}$. In situations of hypoxia, when the oxygen-dependent NO synthases may become dysfunctional, nitrite reduction is instead greatly enhanced. Nitrate and nitrite can thus be viewed as storage pools supporting NO signaling during metabolic stress, with their bioactivation involving both enzymatic and nonenzymatic reactions in blood and tissues. The bioactivation of the more stable nitrate anion involves initial reduction to nitrite by symbiotic bacteria in the oral cavity ${ }^{1}$. A role for nitrite in regulation of blood flow and tissue responses to hypoxia has been reported. In addition, multiple studies now support a therapeutic role for nitrate and nitrite, most notably in the treatment and prevention of cardiovascular diseases including ischemia-reperfusion (IR) injury and hypertension ${ }^{1}$. The nutritional aspects of these cardioprotective effects are particularly intriguing since nitrate and nitrite are abundant in our everyday diet. On June $17^{\text {th }}$

See end for affiliations. and $18^{\text {th }}, 2009$, a 2-day symposium was held at the Nobel Forum, Karolinska Institutet in Stockholm, Sweden to discuss recent advances in understanding the role of nitrate and nitrite in biology, nutrition and therapeutics.

Nitric oxide, nitrite and mitochondria Recent data suggest that many of the biological effects of nitrite ${ }^{6,7}$ involve interaction with mitochondria. In the last 15 years it has been established that the mitochondrion is a physiological target for NO (ref. 8). In the opening keynote lecture, Salvador Moncada (University College London) gave an overview on how NO regulates mitochondrial function in health and disease. NO binds to cytochrome $c$ oxidase, the terminal electron acceptor in the mitochondrial electron transport chain, in competition with oxygen. Moncada reviewed evidence showing that binding of NO to cytochrome $c$ oxidase elicits intracellular signaling events, including the diversion of oxygen to nonrespiratory substrates and the generation of reactive oxygen species (ROS) with potentially

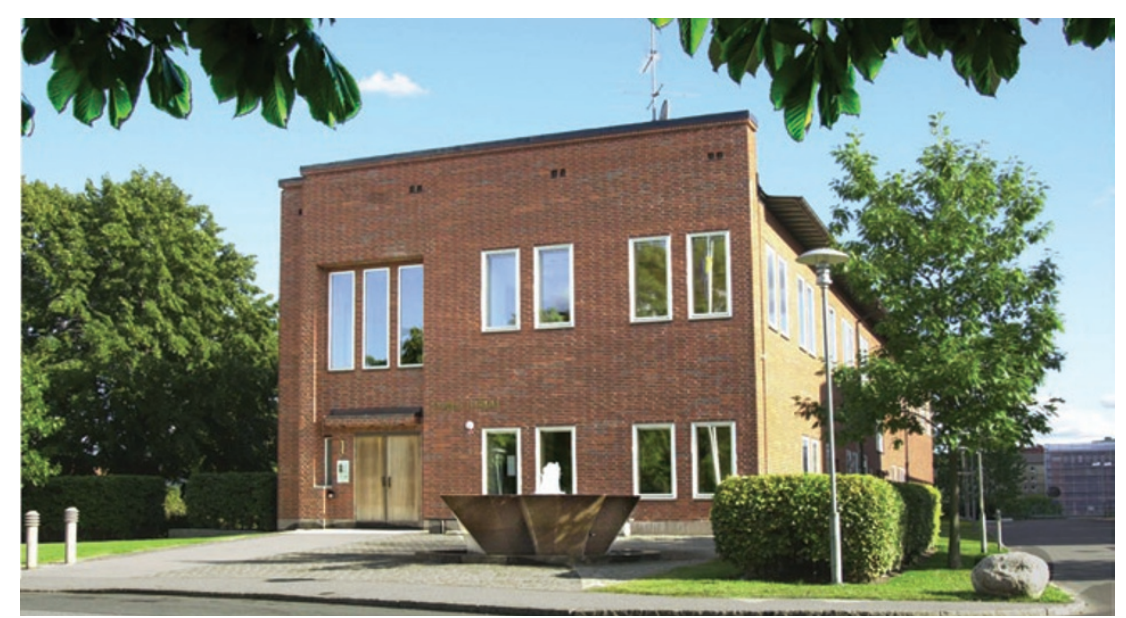

The Nobel Forum at Karolinska Institutet, where the Third International Meeting on the Role of Nitrate and Nitrite in Physiology, Pathophysiology and Therapeutics was held. 


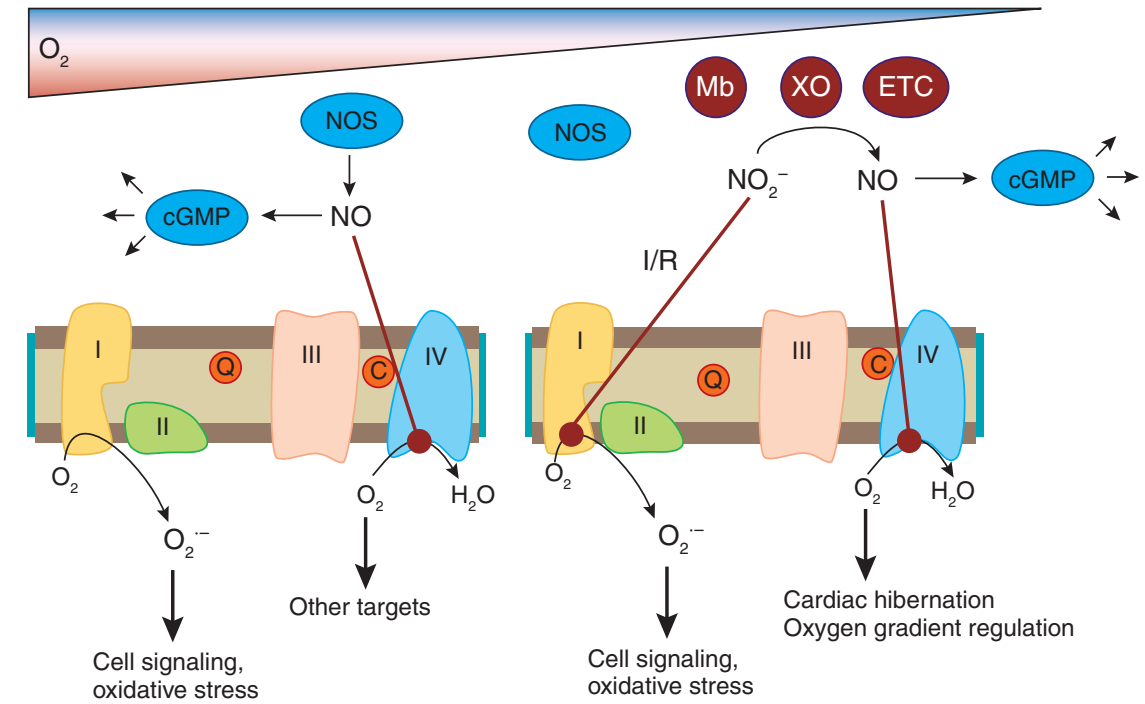

Figure 1 Nitric oxide and nitrite interactions with mitochondria. In normoxic conditions NOS generates NO, which activates cGMP signaling pathways (for example, vasodilation) and directly inhibits complex IV. This allows oxygen to be diverted away from the mitochondria to other targets (such as HIF1 $\alpha$ ). This inhibition also increases ROS generation from the mitochondria, generating increased ROS to potentially mediate cell signaling and oxidative stress. In hypoxia/ischemia, NOS activity is inhibited by the lack of oxygen. Nitrite is reduced to NO by deoxygenated myoglobin (Mb), xanthine oxidoreductase (XO), enzymes of the electron transport chain (ETC) or other enzymes, and the resulting NO can inhibit respiration at complex IV to regulate oxygen gradients or mediate cardiac hibernation. During ischemia/reperfusion, nitrite inhibits complex I via S-nitrosation, which decreases ROS generation at reperfusion, leading to cytoprotection.

damaging effects. He also discussed findings indicating that these $\mathrm{NO}$-elicited events act as triggers by which mitochondria modulate signal transduction cascades involved in the induction of cellular defense mechanisms and adaptive responses. Furthermore, he presented evidence indicating that lack of NO metabolism by cytochrome $c$ oxidase at low oxygen concentrations might explain the phenomenon of hypoxic vasodilatation.

Sruti Shiva (University of Pittsburgh) reviewed a number of studies demonstrating that components of the respiratory chain can reduce nitrite to NO (refs. 4,9), as well as data suggesting that nitrite reduction by myoglobin and xanthine oxidoreductase (XOR) can generate NO during hypoxia that then regulates mitochondrial respiration (Fig. 1). Although the physiological relevance of this is still uncertain, recent data suggest that nitrite-dependent modulation of mitochondrial function may be important in regulating oxygen gradients during hypoxia and mediating cytoprotection after IR injury $^{6}$ (see below).

\section{The biochemistry and bioactivation of nitrite}

Bacteria commonly utilize nitrate and nitrite as terminal electron acceptors for respiration in the absence of oxygen, or for incorporation in biomass. However, it was only recently described that mammalian tissues can also reduce nitrate ${ }^{10}$ and nitrite ${ }^{11}$ to bioactive nitrogen oxides including NO (Fig. 2). It is now clear that the one-electron reduction of nitrite to $\mathrm{NO}$ can be catalyzed by numerous enzymatic as well as nonenzymatic pathways ${ }^{1,4}$. The relative contribution from these pathways to overall NO formation varies between tissues and is dependent on a number of factors including local $\mathrm{pH}$, oxygen tension and redox status.

The ability of deoxygenated hemoglobin and myoglobin to react with and reduce nitrite to NO is currently a matter of intense research, which was reflected by the many presentations on this topic. Tienush Rassaf (University of Dusseldorf) explained that myoglobin-derived NO inhibits cellular respiration and limits ROS formation and mitochondrial enzyme dysfunction after IR injury ${ }^{6,12}$. Nitrite protection against myocardial infarction is lacking in myoglobin knockout mice, which supports the hypothesis that myoglobin serves a critical function as an intrinsic nitrite reductase that regulates cellular responses to hypoxia and reoxygenation ${ }^{13}$.The family of nitritereducing globins has been expanded to include neuroglobin-a monomeric heme protein specifically expressed in the brain and the most ancestral among vertebrate globins ${ }^{14,15}$. Mark Gladwin (University of Pittsburgh) explained that neuroglobin, unlike hemoglobin and myoglobin, has a six-coordinate heme geometry. Such heme geometry greatly limits oxygen binding, raising questions about the physiological functions of these six-coordinate globins. Nevertheless, the expression of neuroglobin is associated with cytoprotective effects in various animal models of brain ischemia. Gladwin showed that if its five-coordinate state is made stable, neuroglobin functions as a very efficient nitrite reductase. He speculated that six-coordinate heme globins in general, including neuroglobin, may function as post-translationally (via a six- to five-coordinate equilibrium) regulated nitrite reductases and primordial NO synthases whose function antedates the evolution of oxygen carriers.

Although many of the biological effects of nitrite may proceed via direct reduction to $\mathrm{NO}$, other bioactive species can also be formed. In addition, it has been suggested that nitrite can signal directly, possibly via nitrosation of thiols and nitrosylation of hemes, without intermediate NO formation ${ }^{16}$. Jack Lancaster (University of Alabama at Birmingham) explained an interesting potential pathway for the generation of nitrosothiols involving NO, nitrate and nitrite reactions with nonheme iron to form dinitrosyl iron complexes ${ }^{17}$. The nitrite anion is also involved in nitration reactions that are commonly implicated in pathological processes such as inflammation and atherosclerosis. Bruce Freeman (University of Pittsburgh) reviewed data on the endogenous formation of nitrated unsaturated fatty acids $\left(\mathrm{NO}_{2}-\mathrm{FA}\right)$ that in turn mediate adaptive and anti-inflammatory signaling reactions. Fatty acid nitration is induced by $\mathrm{NO}$ and nitrite-derived species reacting via multiple mechanisms, ultimately involving homolytic addition of nitrogen dioxide $\left(\mathrm{NO}_{2}\right)$ to the double bond. $\mathrm{NO}_{2}$-FAs display cGMP-independent signaling activity as a consequence of robust electrophilic reactivity, facilitating reversible adduction to nucleophilic targets such as cysteine residues ${ }^{18}$. Overall, these presentations highlighted the function of nitrite as a relatively stable reservoir of $\mathrm{NO}$ bioactivity, which can also undergo secondary reactions with biomolecules to form redoxderived signaling mediators.

\section{Nitrite reduction in the vasculature}

NO-mediated control of blood flow involves direct diffusion of $\mathrm{NO}$ from the primary site of generation in the endothelium. In addition, the red blood cell (RBC) contributes to vasoregulation by exporting NO-like bioactivity as discussed by Jonathan Stamler (Duke University), who originally proposed this mechanism ${ }^{19}$. Different mechanisms for the generation and export of NO bioactivity from the RBC have been proposed ${ }^{14,19,20}$. At this meeting the role 
of nitrite as a source of NO in the circulation was highlighted. Nitrite administration causes vasodilatation at low concentrations ${ }^{14}$, and this is ultimately mediated by activation of cGMP in the vascular smooth muscle. The sites of production and the mechanism for nitrite-dependent NO generation in the vasculature were vigorously discussed, and it was clear that there is still controversy about the significance of endogenous nitrite in physiological regulation of blood vessel tone. Deoxygenated hemoglobin has been implicated in controlling nitrite-dependent NO signaling in the vasculature ${ }^{14,20}$. Rakesh Patel (University of Alabama at Birmingham) discussed the potential mechanisms that may regulate nitrite-RBC interactions, including oxygen fractional saturation-dependent control over nitrite transport into the RBC and hemebased nitrite reduction ${ }^{21}$. Nitrite reduction to NO by deoxygenated hemoglobin may govern $\mathrm{NO}$ signaling in the vasculature as exemplified by experiments showing that nitrite therapy can prevent hypertension induced by cell-free hemoglobin-based oxygen carriers in vivo ${ }^{22}$. A remaining controversial issue is how any $\mathrm{NO}$ formed in the red cell is exported to elicit vasodilatation. Given the extremely fast rate that $\mathrm{NO}$ reacts with hemoglobin, export of free NO from the red blood cell is unlikely. Daniel KimShapiro (Wake Forest University) explained recent data describing formation of the nitrosating agent $\mathrm{N}_{2} \mathrm{O}_{3}$ from the concerted nitrite reductase/anhydrase activity of hemoglobin ${ }^{23}$. Because $\mathrm{N}_{2} \mathrm{O}_{3}$ is less reactive with hemoglobin $(\mathrm{Hb})$ or $\mathrm{HbO}_{2}$ than is $\mathrm{NO}$, this could facilitate export of $\mathrm{NO}$ bioactivity from the $\mathrm{RBC}$, either directly as $\mathrm{N}_{2} \mathrm{O}_{3}$ or via formation of a secondary S-nitrosothiol. Joel Friedman (Albert Einstein College of Medicine) explained how he has been using sol-gel and glassy matrices to dissect the nitrite-hemoglobin interactions on a molecular level ${ }^{24}$. Both Friedman and Pedro Cabrales (University of California) showed data supporting a nitrite reductase and anhydrase activity of hemoglobin as well as hemoglobin-based oxygen carriers. George Richter-Addo (University of Oklahoma) demonstrated, through crystallography, that nitrite binds to the ferric iron center of the protein in an unusual O-binding mode ${ }^{25}$. Interestingly, conformational differences exist in the O-binding of nitrite to the $\alpha$ and $\beta$ subunits of the protein.

Amrita Ahluwalia (Barts and The London Medical School) presented data demonstrating expression of eNOS and XOR on the membranes of human RBCs and blood vessels ${ }^{26}$. By using inhibitors of XOR and NO synthase, Ahluwalia suggested that the nitrite reductase activity within the circulation (under hypoxic conditions and physiological $\mathrm{pH}$ ) is mediated in part by eNOS, while a substantial role for XOR

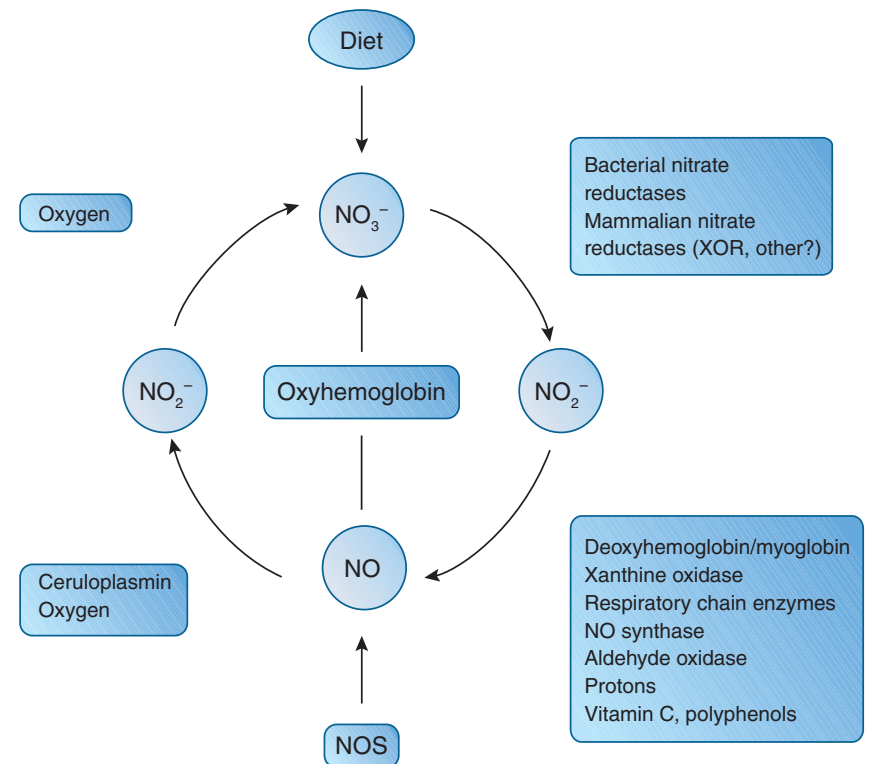

Figure 2 A mammalian nitrogen oxide cycle. Nitric oxide synthase (NOS) generates NO in cells to regulate a vast variety of physiological functions. The bioactivity of NO is acutely terminated by its rapid oxidation to nitrite and nitrate. In our bodies nitrate can undergo reduction to nitrite $\left(\mathrm{NO}_{2}{ }^{-}\right)$by bacteria in the oral cavity and by xanthine oxidase and possibly other enzymes in tissues. In blood and tissues nitrite can be further metabolized to nitric oxide (NO) and other biologically active nitrogen oxides (not shown in figure). This reduction is catalyzed by various enzymatic and nonenzymatic pathways, most of which are greatly enhanced under hypoxic conditions. The reduction of nitrate and nitrite to NO completes a mammalian nitrogen oxide cycle.

becomes evident as acidosis develops. Further studies are required to determine exactly when and where XOR is recruited to act as a nitrite reductase in vivo. Jay Zweier (Ohio State University) discussed the mechanisms for nitrite reduction in the vessel wall. NO formation was detected by electrochemical and electron paramagnetic resonance techniques in isolated vessels that dilated following nitrite treatment. These events could be blocked by adding either hemoglobin or an inhibitor of soluble guanylyl cyclase (sGC) ${ }^{27}$. Zweier suggested that sGC or a resident heme protein might catalyze nitrite reduction to NO.

\section{Dietary nitrate in health and disease}

Our everyday diet is a major source of nitrate, and the levels are particularly high in certain vegetables. As an example, one serving of spinach, lettuce or beetroot contains more nitrate than that generated endogenously over a day by all three NOSs combined ${ }^{1}$. A substantial amount of ingested nitrate is actively taken up from the circulation by the salivary glands and concentrated in saliva. Salivary nitrate is then efficiently reduced to nitrite by bacteria in the oral cavity, leading to 1,000-fold higher concentrations of nitrite in saliva compared to plasma $^{28}$. The continuous swallowing of nitritecontaining saliva ( $\sim 1.5$ liters per day) generates a variety of nitrogen oxides, including $\mathrm{NO}$, in the acidic milieu of the gastric lumen. Actually, the initial discoveries that nitrate and nitrite can be converted to $\mathrm{NO}$ in mammals came from observations that the gastric lumen contains high levels of NO (refs. 29,30). The enterosalivary circulation of nitrate also leads to a sustained increase in plasma levels of nitrite ${ }^{31}$, and nitrate may therefore be considered as a 'prodrug' of nitrite with a slow-release profile.

Over the past half century, dietary nitrate and nitrite have become notorious because of their supposed association with the development of disease, most notably gastric cancer ${ }^{32}$. This has led to strict regulations of nitrate and nitrite levels in food and drinking water. Kenneth McColl (Glasgow University) described the potential harmful effects of nitrate-derived gastric NO. The generation is maximal at the gastroesophageal junction where salivary nitrite first meets gastric acid ${ }^{33}$. The NO formed diffuses into the tissue where it forms nitrosating species, which in turn may cause DNA damage and promote carcinogenesis. According to McColl ascorbic acid would paradoxically further aggravate the carcinogenic effects since this vitamin greatly enhances NO formation from acidified nitrite. In the same session Nigel Benjamin (University of Exeter and Plymouth) surveyed the underlying epidemiological evidence for the recommended limitations of nitrate and nitrite in food and drinking water. He concluded that there is relatively weak evidence to support the present limitations ${ }^{34}$, especially in light of the 
beneficial effects of nitrate and nitrite that have emerged during recent years. Interestingly, a diet rich in vegetables, such as the Mediterranean and the traditional Japanese diets, contains more nitrate than that recommended as the acceptable daily intake (ADI) by the World Health Organization ${ }^{34}$. Joel Petersson (Uppsala University) discussed physiological and therapeutic NO-dependent effects of dietary nitrate in the gastrointestinal tract. Salivary nitrite increases gastric mucosal blood flow and mucus production, thereby supporting gastroprotective mechanisms ${ }^{35}$.

A diet rich in fruit and vegetables reduces blood pressure and protects against cardiovascular disease, but the active ingredients responsible for these effects have not been pinpointed. Recently it was shown that modest dietary supplementation with sodium nitrate reduces blood pressure in healthy volunteers ${ }^{36}$. Andrew Webb (Barts and The London Medical School) and colleagues used beetroot juice as a source of nitrate in healthy volunteers and found a reduction in blood pressure of $\sim 10$ $\mathrm{mm} \mathrm{Hg}$ that coincided with peak plasma levels of nitrite ${ }^{37}$. In addition, inhibitory effects on ex vivo platelet aggregation and protection against IR-induced endothelial dysfunction were observed. Similar data were presented by Mattias Carlström (Uppsala University), who observed a sustained effect on blood pressure by chronic dietary nitrate supplementation in rats ${ }^{38}$. Both groups could prevent the effects on blood pressure either by letting the human volunteers spit for $3 \mathrm{~h}$ or by application of an antibacterial mouthwash to the rats. This shows the importance of the enterosalivary circulation of nitrate and the critical role of oral nitratereducing bacteria in its bioactivation. Nathan Bryan (University of Texas) argued that nitrate and nitrite should be viewed as bioactive food components and indispensable nutrients that may serve to uphold $\mathrm{NO}$ /nitroso homeostasis, especially in conditions where the L-arginineNOS pathway is dysfunctional. Bryan explained that dietary nitrate and nitrite protect against IR injury in experimental myocardial infarction ${ }^{39}$; and in mice fed a cholesterol-enriched diet, the addition of nitrite to the drinking water prevented the signs of vascular pathology $y^{40}$.

\section{Nitrate, nitrite and energetics}

The role of NO in regulation of mitochondrial function and energy expenditure has attracted much attention. Recently it was demonstrated that dietary nitrate supplementation reduces oxygen cost and improves muscular efficiency at various levels of submaximal exercise in healthy young volunteers without increasing anaerobic metabolism ${ }^{7}$. Andy Jones (University of Exeter) presented data demonstrating that in healthy volunteers given a dietary nitrate load (in the form of beetroot juice), oxygen consumption was reduced, resulting in a concomitant improvement of performance ${ }^{41}$. The mechanisms underlying these remarkable effects of dietary nitrate need further investigation, and the discussion at the meeting focused on effects at the mitochondrial level. Similar mechanisms may be at work when nitrite and nitrate are produced from endogenous NO. To this end, Martin Feelisch (University of Warwick) presented preliminary results from an ongoing study in mountaineers, who experience an integrated whole-body response to exercise and hypoxia. While ascent to altitude was associated with enhanced oxidative stress and discrete increases in circulating nitrite and nitrate, no simple relationship of these "NO markers" to the prevailing level of hypoxia was apparent, which suggests that the in vivo situation is complex. It is established that exercise provides protection against cardiovascular disease, and David Lefer (Emory University School of Medicine) showed data on exercise-induced increases in circulating and myocardial nitrite and S-nitrosothiol levels in mice. The increase in myocardial storage of nitrite after exercise was associated with protection during acute myocardial infarction.

\section{Therapeutic opportunities}

In recent years data have accumulated showing that nitrite protects against IR injury in the heart, brain, kidney and liver, as well as in chronic limb ischemia (for review see refs. 1,2). At the meeting a number of new therapeutic indications were discussed. In their presentations Brian Zuckerbraun (University of Pittsburgh) and Erik Swenson (University of Washington) focused on the pulmonary effects of nitrite where both inhalation therapy and intravenous administration were shown to be protective in models of pulmonary hypertension, ventilatorinduced lung injury and lung IR injury.

Renal injury is a common complication in people with hypertension. Koichiro Tsuchiya (University of Tokushima) presented data showing that very low doses of dietary nitrite attenuated proteinurea and prevented the renal histological damage induced by chronic administration of an NOS inhibitor ${ }^{42}$. These data indicate that the nitrate-nitrite-NO pathway can compensate for disturbances in endogenous NO generation from NOSs.

John Lang (University of Washington) and Kenneth McCurry (Cleveland Clinic) discussed ongoing studies on nitrite therapy during solid organ transplantation. They demonstrated beneficial effects of nitrite in animal models of liver and heart transplantation, respectively. Administration of nitrite to the organ preservation fluid and to the organ recipient improved parameters of organ survival and function ${ }^{43}$.

In aggregate, promising data are emerging in a wide variety of conditions predominantly involving tissue ischemia. This insight is catalyzing the evaluation of potential therapeutic benefits of nitrate and nitrite administration, in the context of L-arginine-NO signaling. A motivation for this is the limited success realized when therapeutic trials have been pursued for clinical indications using clinically prevalent NO donors and inhibitors of NO synthases. This could be due to the ubiquitous presence of different NOS isoforms, the pluripotency of $\mathrm{NO}$ and the rather nonselective release of $\mathrm{NO}$ from most donors. In contrast, nitrite bioactivation is promoted in anatomic regions undergoing ischemia/hypoxia, leading to potentially more localized therapeutic effects. Another possible advantage is the apparent absence of tolerance connected to relatively long-term nitrite ${ }^{44}$ or nitrate ${ }^{38}$ administration in contrast to treatment with organic nitrates such as nitroglycerin.

\section{Conclusions and future perspectives}

During a rather short time span our insight into the nitrate-nitrite-NO pathway has improved dramatically. Now, future research challenges include addressing a range of critical issues. Perhaps the most important is determining the physiological roles of endogenous nitrate and nitrite levels. This is not trivial, however, since in contrast to NOS-dependent physiology, which has been largely explored by the use of NOS inhibitors, there is no specific nitrite reductase inhibitor available. Moreover, the dual origin of nitrate and nitrite (from our diet and from endogenous production) presents a vexing obstacle in experimental design.

The mechanisms accounting for nitrite bioactivation clearly will include new candidate enzymes that may be specific for different tissues and/or at different $\mathrm{pH}$ or oxygen gradients. Moreover, it is important to understand the mechanisms and compartments within the vasculature where nitrite reduction occurs and the roles of various physiological $\mathrm{pH}$ and oxygen tension extremes in the generation of various metabolites. Also, a greater appreciation is needed of how endogenous levels of nitrate and nitrite are regulated and transported and how they interface with the NOS pathway.

This report reveals new perspectives on the therapeutic use of nitrate and nitrite that are actively emerging and consequently setting the stage for future clinical trials related to conditions involving ischemia and reperfusion. The nutritional aspects of the nitrate-nitriteNO pathway are also particularly intriguing 
and will motivate a vibrant discussion challenging the dogma that dietary nitrate and nitrite are harmful. Of crucial relevance, the salutary effects of nitrate and nitrite that are emerging may also explain some of the beneficial health effects of a diet rich in vegetables, such as the Mediterranean diet, thereby instigating a discussion of the revision of acceptable levels of intake.

In summary, future laboratory, clinical and epidemiologic investigations of the role of nitrate and nitrite in physiology, pathophysiology, nutrition and therapeutics promise to bear fruit regarding the development of new therapeutic avenues for important clinical problems and can shift current views on our dietary constituents in the context of health and disease.

\section{ACKNOWLEDGMENTS}

This meeting was generously funded by the Department of Physiology and Pharmacology at the Karolinska Institutet, the Vascular Medicine Institute (supported by funds from the Hemophilia Center of Western Pennsylvania and the Institute for Transfusion

Medicine) and the Department of Pharmacology and Chemical Biology at the University of Pittsburgh. The meeting was also sponsored by Hope Pharmaceuticals and Ikaria. We are grateful to Elsevier and Nitric Oxide: Biology and Chemistry for kindly printing the meeting abstract book. In addition, we acknowledge generous support from Vinnova (CIDaT), the European Union's 7th Framework Program (FLAVIOLA), and Torsten and Ragnar Söderbergs Foundation. Additional information on speakers and presentations can be found at http:// www.strategicresults.com/nitrite/.

\section{COMPETING INTERESTS STATEMENT}

The authors declare competing financial interests: details accompany the full-text HTML version of the paper at http://www.nature.com/ naturechemicalbiology/.

1. Lundberg, J.O., Weitzberg, E. \& Gladwin, M.T. Nat. Rev. Drug Discov. 7, 156-167 (2008).

2. Gladwin, M.T. et al. Nat. Chem. Biol. 1, 308-314 (2005).

3. Reutov, V.P. \& Sorokina, E.G. Biochemistry (Mosc.) 63, 874-884 (1998)

4. van Faassen, E.E. et al. Med. Res. Rev. 29, 683-741 (2009).

5. Weitzberg, E. \& Lundberg, J.O. Nitric Oxide 2, 1-7 (1998).

6. Shiva, S. et al. J. Exp. Med. 204, 2089-2102 (2007).

7. Larsen, F.J., Weitzberg, E., Lundberg, J.O. \& Ekblom, B. Acta Physiol. (Oxf.) 191, 59-66 (2007).

8. Erusalimsky, J.D. \& Moncada, S. Arterioscler. Thromb. Vasc. Biol. 27, 2524-2531 (2007).

9. Kozlov, A.V., Staniek, K. \& Nohl, H. FEBS Lett. 454 , 127-130 (1999).

10. Jansson, E.A. et al. Nat. Chem. Biol. 4, 411-417 (2008).

11. Zweier, J.L., Wang, P., Samouilov, A. \& Kuppusamy, P. Nat. Med. 1, 804-809 (1995)

12. Rassaf, T. et al. Circ. Res. 100, 1749-1754 (2007).

13. Hendgen-Cotta, U.B. et al. Proc. Natl. Acad. Sci. USA 105, 10256-10261 (2008)

14. Cosby, K. et al. Nat. Med. 9, 1498-1505 (2003).

15. Petersen, M.G., Dewilde, S. \& Fago, A. J. Inorg. Biochem. 102, 1777-1782 (2008).

16. Bryan, N.S. et al. Nat. Chem. Biol. 1, 290-297 (2005).

17. Bosworth, C.A., Toledo, J.C. Jr. Zmijewski, J.W., Li, Q. \& Lancaster, J.R. Jr. Proc. Natl. Acad. Sci. USA 106, 4671-4676 (2009).

18. Freeman, B.A. et al. J. Biol. Chem. 283, 15515-15519 (2008).

19. Jia, L., Bonaventura, C., Bonaventura, J. \& Stamler, J.S. Nature 380, 221-226 (1996)

20. Nagababu, E., Ramasamy, S., Abernethy, D.R. \& Rifkind,
J.M. J. Biol. Chem. 278, 46349-46356 (2003).

21. Vitturi, D.A. et al. Am. J. Physiol. Heart Circ. Physiol. 296, H1398-H1407 (2009).

22. Rodriguez, C. et al. Biochem. J. 422, 423-432 (2009).

23. Basu, S. et al. Nat. Chem. Biol. 3, 785-794 (2007).

24. Roche, C.J., Dantsker, D., Samuni, U. \& Friedman, J.M. J. Biol. Chem. 281, 36874-36882 (2006).

25. Yi, J., Safo, M.K. \& Richter-Addo, G.B. Biochemistry 47 8247-8249 (2008)

26. Webb, A.J. et al. Circ. Res. 103, 957-964 (2008)

27. Alzawahra, W.F., Talukder, M.A., Liu, X., Samouilov, A. \& Zweier, J.L. Am. J. Physiol. Heart Circ. Physiol. 295, H499-H508 (2008).

28. Lundberg, J.O., Weitzberg, E., Cole, J.A. \& Benjamin, N. Nat. Rev. Microbiol. 2, 593-602 (2004).

29. Lundberg, J.O., Weitzberg, E., Lundberg, J.M. \& Alving, K. Gut 35, 1543-1546 (1994).

30. Benjamin, N. et al. Nature 368, 502 (1994).

31. Lundberg, J.0. \& Govoni, M. Free Radic. Biol. Med. 37 395-400 (2004).

32. Tannenbaum, S.R. \& Correa, P. Nature $\mathbf{3 1 7}, 675-676$ (1985).

33. lijima, K. et al. Gastroenterology 122, 1248-1257 (2002).

34. Alexander, J. et al. EFSA J. 689, 1-79 (2008).

35. Bjorne, H.H. et al. J. Clin. Invest. 113, 106-114 (2004).

36. Larsen, F.J., Ekblom, B., Sahlin, K., Lundberg, J.O. \& Weitzberg, E. N. Engl. J. Med. 355, 2792-2793 (2006).

37. Webb, A.J. et al. Hypertension 51, 784-790 (2008).

38. Petersson, J. et al. Free Radic. Biol. Med. 46, 10681075 (2009).

39. Bryan, N.S. et al. Proc. Natl. Acad. Sci. USA 104 19144-19149 (2007)

40. Stokes, K.Y. et al. Am. J. Physiol. Heart Circ. Physiol. 296, H1281-H1288 (2009).

41. Bailey, S.J. et al. J. Appl. Physiol. 107, 1144-1155 (2009).

42. Kanematsu, Y. et al. Am. J. Physiol. Renal Physiol. 295 F1457-F1462 (2008).

43. Zhan, J. et al. Surgery 146, 155-165 (2009).

44. Dejam, A. et al. Circulation 116, 1821-1831 (2007).

Jon O. Lundberg is in the Department of Physiology and Pharmacology, Karolinska Institutet, Stockholm, Sweden. Mark T. Gladwin and Sruti Shiva are in the Pulmonary, Allergy and Critical Care Medicine and Vascular Medicine Institute, University of Pittsburgh, Pittsburgh, Pennsylvania, USA. Amrita Ahluwalia and Andrew J. Webb are in the Centre for Clinical Pharmacology at the William Harvey Research Institute, Barts and the London School of Medicine and Dentistry, Queen Mary University of London, London, UK. Nigel Benjamin is at the University of Exeter and Plymouth, Exeter, UK. Nathan S. Bryan is in the Brown Foundation Institute of Molecular Medicine, The University of Texas Health Science Center at Houston, Houston, Texas, USA. Anthony Butler is at the University of St. Andrews, Fife, Scotland, UK. Pedro Cabrales is in the Department of Bioengineering, University of California San Diego, La Jolla, California, USA. Angela Fago is in the Department of Biological Sciences, Aarhus University, Aarhus, Denmark. Martin Feelisch is in the Warwick Medical School at The University of Warwick, Coventry, UK. Peter C. Ford is in the Department of Chemistry and Biochemistry, University of California Santa Barbara, Santa Barbara, California, USA. Bruce A. Freeman is in the Department of Pharmacology and Chemical Biology, University of Pittsburgh School of Medicine, Pittsburgh, Pennsylvania, USA. Michael Frenneaux is at the University of Birmingham, Birmingham, UK. Joel Friedman is in the Albert Einstein College of Medicine, Bronx, New York, USA. Malte Kelm and Tienush Rassaf are at the University of Dusseldorf, Dusseldorf, Germany. Christopher G. Kevil is in the Department of Pathology, Louisiana State University Health Sciences Center, Shreveport, Louisiana, USA. Daniel B. Kim-Shapiro is in the Department of Physics, Wake Forest University, Winston-Salem, North Carolina, USA. Andrey V. Kozlov is in the Ludwig Boltzmann Institute for Experimental and Clinical Traumatology, Vienna, Austria. Jack R. Lancaster Jr. and Rakesh P. Patel are at the University of Alabama Birmingham, Birmingham, Alabama, USA. David J. Lefer is at the Emory University School of Medicine, Atlanta, Georgia, USA. Kenneth McColl is at the University of Glasgow, Glasgow, Scotland, UK. Kenneth McCurry is in the Cleveland Clinic, Cleveland, Ohio, USA. Joel Petersson is in the Department of Medical Cell Biology, Uppsala University, Uppsala, Sweden. George B. Richter-Addo is in the Department of Chemistry and Biochemistry, University of Oklahoma, Norman, Oklahoma, USA. Valentin P. Reutov is in the Institute of Higher Nervous Activity and Neurophysiology, Russian Academy of Sciences, Moscow, Russia. Alan Schechter is in the National Institute of Diabetes and Digestive and Kidney Diseases, US National Institutes of Health, Bethesda, Maryland, USA. Koichiro Tsuchiya is in the Institute of Health Biosciences at the University of Tokushima, Tokushima, Japan. Ernst E. van Faassen is at the University of Utrecht, Utrecht, The Netherlands. Brian S. Zuckerbraun is at the University of Pittsburgh School of Medicine, Pittsburgh, Pennsylvania, USA. Jay L. Zweier is in the Departments of Medicine, Physiology and Biochemistry, The Ohio State University, Columbus, Ohio, USA. Eddie Weitzberg is in the Department of Physiology and Pharmacology, Section for Anesthesiology and Intensive Care Medicine, Karolinska Institutet, Stockholm, Sweden.

e-mail:jon.lundberg@ki.seoreddie.weitzberg@ki.se 